\title{
Status of the Fast Interaction Trigger detector for the ALICE upgrade
}

\author{
Solangel Rojas-Torres ${ }^{a, *}$ for the ALICE Collaboration \\ ${ }^{a}$ Czech Technical University in Prague, \\ Brehova 7, Prague, Czech Republic \\ E-mail: solangel.rojas.torres@cern.ch, rojassol@fjfi.cvut.cz
}

During the second Long Shutdown (LS2) of the LHC, ALICE has installed three new detectors and implemented continuous data readout with online reconstruction and data compression for several other subsystems. The changes are needed to benefit from the increased luminosity of the LHC during Run 3 and 4. The ALICE interaction rate will increase, with respect to Run 1 and 2, by up to two orders of magnitude, reaching $50 \mathrm{kHz}$ for $\mathrm{Pb}-\mathrm{Pb}$ and up to $1 \mathrm{MHz}$ for pp collisions. One of the new ALICE detectors is the Fast Interaction Trigger (FIT). Its main functionality includes generating minimum-latency interaction triggers $(<425 \mathrm{~ns})$, luminosity monitoring with online feedback to the LHC, a precise measurement of the collision time with a resolution better 7 than $40 \mathrm{ps}$, determination of the centrality and event plane for heavy-ion collisions, and tagging of diffractive and ultra-peripheral events. The FIT detector consists of three subsystems: two fast Cherenkov arrays with $2 \mathrm{~cm}$ thick quartz radiators coupled to modified MCP-PMT photosensors (FT0), a large-area scintillator disc (FV0) implementing a novel light collection system, and the forward diffractive detector (FDD). FDD comprises two plastic scintillator arrays with fast wavelength shifting bars, optical fibre bundles, and PMTs. The FDD arrays are located $\pm 20 \mathrm{~m}$ away from the nominal interaction point, along the beam line. A brief description of the detector and its functionalities is given together with the installation and commissioning status.

\footnotetext{
*** The European Physical Society Conference on High Energy Physics (EPS-HEP2021), ***

*** 26-30 July $2021 * * *$

*** Online conference, jointly organized by Universität Hamburg and the research center DESY ***
}

\footnotetext{
${ }^{*}$ Speaker
} 
ALICE (A Large Ion Collider Experiment) [1] is dedicated to the study of strongly-interacting matter at high temperature and energy density produced in ultra-relativistic collisions of heavy ions at the LHC (Large Ion Collider) at CERN. ALICE is currently completing global commissioning before the February 2022 start of the LHC Run 3. To take advantage of the increase in the collision rate and luminosity up to $50 \mathrm{kHz}$ and $6 \times 10^{-27} \mathrm{~cm}^{2} s^{-1}$ for $\mathrm{Pb}-\mathrm{Pb}$; and $1 \mathrm{MHz}$ and $10^{-34} \mathrm{~cm}^{-2} \mathrm{~s}^{-1}$ for pp collisions, ALICE has installed three new detectors [2]: the Inner Tracking System (ITS), the Muon Forward Tracking (MFT) and the Fast Interaction Trigger (FIT) [3]. ALICE has also upgraded other detectors, including the Time Projection Chamber (TPC) and developed the new Online-Offline $(\mathrm{O} 2)$ software infrastructure to handle both triggered and continuous readout of the sensors. In addition to providing minimum-latency triggers ( $<425 \mathrm{~ns})$ to the ALICE Central Trigger Processor, FIT will also monitor luminosity and feed it directly to the LHC. In the offline mode, FIT will measure the collision time with a resolution better than $40 \mathrm{ps}$, determine the centrality and event plane for heavy-ion collisions, and tag diffractive and photon-induced processes [4].

\section{The design of FIT}

FIT is composed of three devices, called FT0, FV0 and FDD. They are located in the forward direction of ALICE and cover almost 8 pseudorapidity units. FT0 and FDD are two-arm detectors where its two components are located at either side of the nominal interaction point along the beam direction. FV0 has only one component. All the detector systems of FIT are shown in Figure 1. They employ different construction strategies and share a common detector-control system (DCS); front-end electronics (FEE) — capable of running both in trigger, and in continuous readout modes; and a laser-calibration system (LCS). Nevertheless, the three detectors use different technologies to accomplish their proposes, as is explained further in this document.

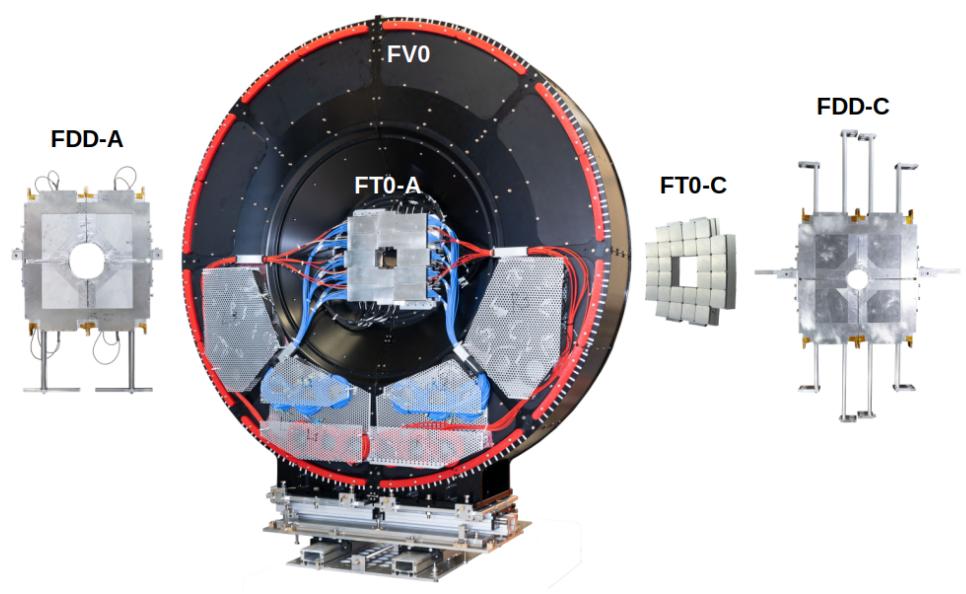

Figure 1: Fast Interaction Trigger components, from left: FDD-A, FT0-A, FV0, FT0-C and FDD-C.

\subsubsection{FT0}

The FT0 sub-detector is composed of two arrays, FT0-A and FT0-C -the A and C ending names are taken from the LHC convention, where the $\mathrm{A}$ and $\mathrm{C}$ side refers to the positive and 
negative directions with respect to the interaction point in the beam direction axis- of custom-made four-pixel micro-channel plate photomultiplier tubes (MCP-PMT) [5] with a $2 \mathrm{~cm}$ thick Cherenkov quartz-radiator attached to each MCP-PMT pixel, as is shown in the left picture of Figure 2.
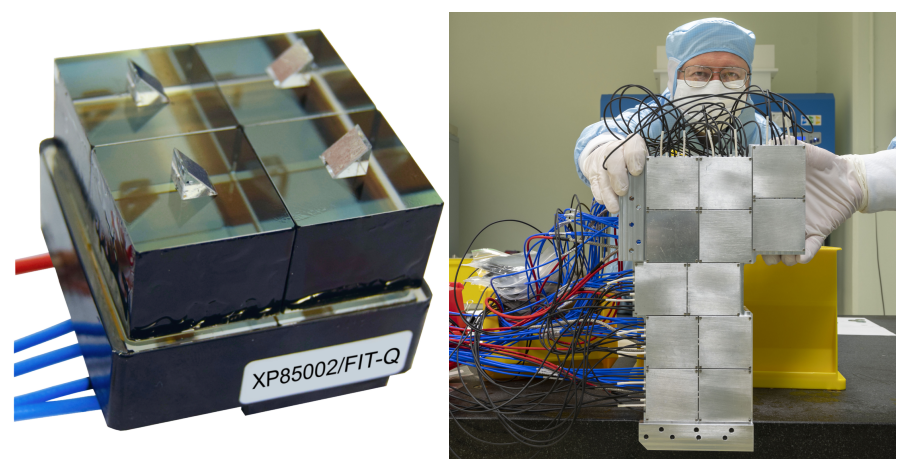

Figure 2: Left: photograph of an MCP-PMT sensor with four quartz radiators coupled to the front window. The tiny prisms are used to deflect LCS light to the photocathode. Right: half of the FT0-A array after assembly at CERN.

The FT0-A (FT0-C) array is placed at $3.3 \mathrm{~m}(-0.819 \mathrm{~m})$ from the interaction point, with a pseudorapidity coverage of $3.5<\eta<4.9(-3.3<\eta<-4.9)$ and has 24 (28) modules and a total of 96 (112) readout channels.

Due to the need for a compact detector, and the required radiation tolerance and magneticfield sensitivity, FT0 uses the MCP-PMT model XP85002/FIT-Q manufactured by Planacon. This particular model was selected from the comparison of several candidates. Then the device was customised to improve its performance; e.g., to avoid cross-talk between the MCP-PMT pixels, increase the dynamic range, improve the time resolution, etc. All the MCP-PMTs where tested individually to optimise their placement according to the workload expected based on simulations. This is really important for the FT0-C, because there will not be access to the detector during the entire Run 3. This system has a time resolution better than $50 \mathrm{ps}$ for a single minimum ionisation particle. This resolution is improved for the case of larger amplitudes or by multi-channel averaging.

\subsubsection{FV0}

FV0 is a plastic scintillator detector with an inner and outer radius of $8 \mathrm{~cm}$ and $144 \mathrm{~cm}$, respectively. It is segmented in 5 concentric rings. The outer ring is divided in 16 azimuth sectors, while the other four rings are divided in 8 . In total FV0 has 48 readout channels that use a novel light collection with optical fibres [6], which consist in attaching one end of the optical fibre facing the plastic scintillator and the other end facing the PMT window, as is shown in Figure 3. These fibres are distributed equidistantly over all the area of the scintillator to ensure a uniform response across the plastic scintillator area, a time resolution around 200-250 ps, and a signal width inside the 25 ns LHC-clock time widow.

As FV0 is placed inside the ALICE L3-magnet, it was required to use fine-mesh PMTs (model H6614-70-Y001) for readout. This detector is placed 3.16 meters from the interaction point and its large area allows it to cover the $2.2<\eta<5.0$ region. Its large angular acceptance makes it an important detector for the determination of the collision plane and the centrality of the collisions. 


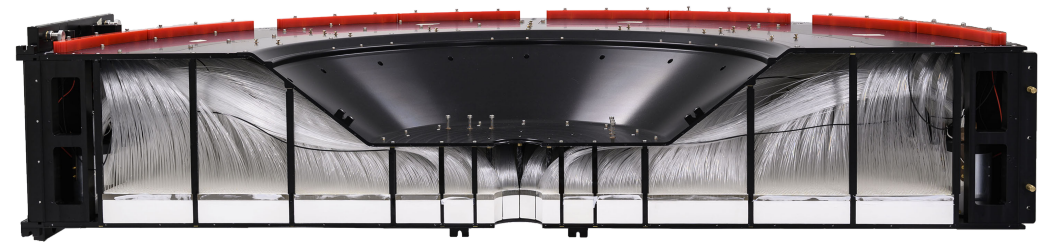

Figure 3: Photograph of one FV0 half with the inner side covers removed.

\subsubsection{FDD}

The forward diffractive detector (FDD) [7] is the upgrade of the former AD detector [8]. It is made of two arrays of plastic scintillator pads. Each array has eight pads assembled in two layers of four pads each for a total of 16 readout channels. The pads are made of $21 \times 18 \times 2.5$ $\mathrm{cm}^{3}$ plastic scintillators with a cut in one of the corners to surround the beam pipe when it is assembled (see Figure 4); each plastic scintillator (Bicron BC-420) has two fast wavelength-shifting (WLS) bars of 1 ns re-emission time (manufactured by Bicron and doped with NOL-38) attached to the sides and optical fibre bundles to transport the light from the WLS bars to fine-mesh PMTs (Hamamatsu H8409-70).

The two arrays, FDD-A and FDD-C, are placed at $17 \mathrm{~m}$ and $-19 \mathrm{~m}$, respectively, from the nominal interaction point. They cover $4.8<\eta<6.3$ and $-7.0<\eta<-4.9$. FDD-A is placed inside the ALICE cavern, while FDD-C is in the LHC tunnel, close to the wall that separate the tunnel from the cavern. Due to space constraints the fibre bundles have different lengths: $1 \mathrm{~m}$ and $0.57 \mathrm{~m}$ for the FDD-A bottom and top halves respectively, and $3 \mathrm{~m}$ for the FDD-C in order to transport the light from the tunnel where the pads are installed to the PMTs that are located inside the ALICE cavern.

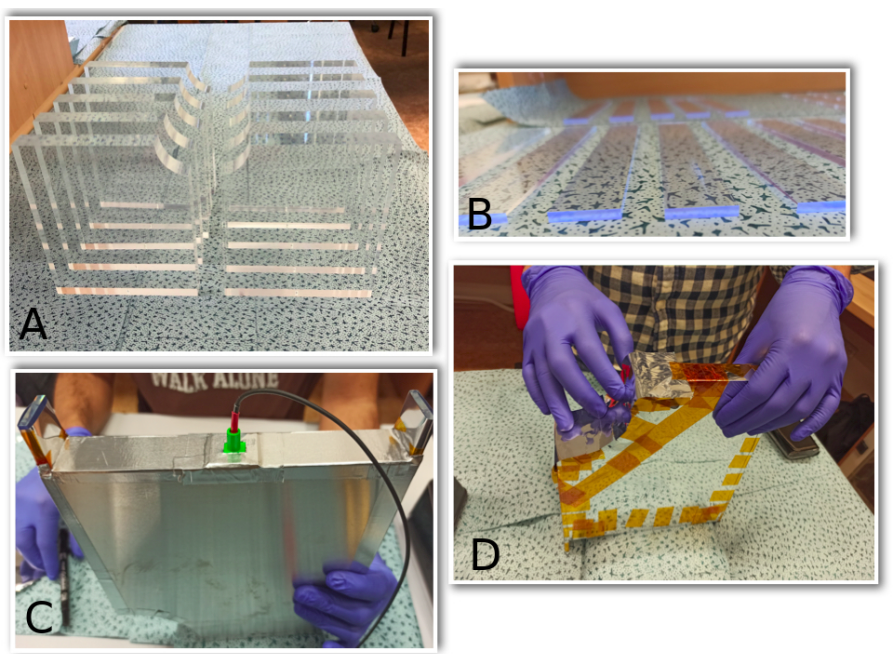

Figure 4: Components and different stages in the assembly of the FDD modules: A) plastic scintillators, B) wavelength shifting bars, C) fully wrapped FDD module with the laser calibration connection port shown in green, and D) first wrapping of the detector with aluminum foil. 


\section{Construction and installation}

The FIT Collaboration is formed by sixty-four scientists representing 17 institutions from Austria, Czech Republic, Denmark, Finland, Mexico, Poland, Russia, and the United States. The FIT detectors are expected to serve ALICE until the end of Run 4.

The sequence followed for the installation of the FIT sub-detectors was determined by the logistics to install other ALICE detectors, and their services as well as by the constraints imposed by the LHC program to access the LHC tunnel.

The FT0-C detector was the first detector to be installed. It was assembled at CERN and then integrated in a common mechanical structure with the Muon Forward Tracker [9]. Due to the complexity of the installation because of the proximity to the beam pipe, the procedure was first validated with a real size mock-up in a meticulous rehearsal on November 25th, 2020 and then successfully inserted and installed in the central barrel of ALICE on December 3rd, 2020.

The FDD-C array was installed next in the LHC tunnel in the RB26 sector, just outside the ALICE cavern. For the installation of the FDD-C a short time window was granted at the end of February 2021, due the restrictions for working in this sector. First, the FDD-C scintillators array was installed in the LHC tunnel and then the optical fibres were passed through a passage from the ALICE cavern to be attached and fixed to the WLS bars.

The mechanical structure of FV0 consists of two half-discs that can be slid and closed around the beam pipe for installation. This mechanical structure was also designed to integrate FT0-A to it. The two halves of FT0-A were fixed to the corresponding FV0 half and installed together in the ALICE cavern on June 21st, 2021. During the installation, due to the strict space constraints, the FV0 and FT0-A were placed in rails for the insertion and then rotated to close around the beam pipe. The full procedure took around 12 hours to be completed, to avoid damage to the beam pipe, which is very close to the detector. Additionally a beam-pipe protection was installed to avoid any accidental damage.

The last FIT sub-detector being installed in ALICE was FDD-A. The procedure had to wait until the installation and baking of the beam pipe was completed. FDD-A was attached to the cavern wall on July 14th, 2021.

The design and construction of the front-end electronics and readout were among the major challenges. FIT electronics is based on just two custom-made units: 1) the processing module (PM), responsible for charge and time digitisation, and 2) the trigger and clock module (TCM) that generates the trigger and communicates with the ALICE central trigger processor and the detector control system. The boards, handling over 300 input signals from the FIT photosensors, are installed in a modified LHC crate. Signal processing and trigger generation is fully digital and is accomplished in just $200 \mathrm{~ns}$.

\section{Final comments}

At the time of the writing of this document, the FEE and all FIT sub-detectors are completely installed in the ALICE site. The integration of the detector control system, readout and onlineoffline software to the global system of ALICE is in progress, being tested, calibrated and fine tuned 
to take data in the first LHC pilot beam tests at the end of October of 2021.

Acknowledgements This research was partially funded by the Ministry of Education, Youth and Sports of the Czech Republic grant number LM2018104.

\section{References}

[1] ALICE Collaboration, Upgrade of the ALICE Experiment: Letter Of Intent. J. Phys. G41, 2014. doi.org/10.1088/0954-3899/41/8/087001.

[2] W.H. Trzaska, New ALICE detectors for Run 3 and 4 at the CERN LHC. NIM A 958(2020)162116. https://doi.org/10.1016/j.nima.2019.04.070

[3] Trzaska, W. H. for the Alice Collaboration, New Fast Interaction Trigger for ALICE. Nuclear Instruments and Methods in Physics Research Section A, vol. 845, 2017. doi.org/10.1016/j.nima.2016.06.029.

[4] Contreras, J.G. and Tapia Takaki, J.D., Ultra-peripheral heavy-ion collisions at the LHC. Int. J. Mod. Phys. A 30, 1542012 (2015). doi.org/10.1142/S0217751X15420129.

[5] Yu.A. Melikyan et al., Performance of the cost-effective Planacon ${ }^{\circledR}$ MCP-PMTs in strong magnetic fields, Nuclear Instruments and Methods in Physics Research Section A: vol. 983, p. 164591, 2020. doi.org/10.1016/j.nima.2020.164591.

[6] Grabski Varlen, New fiber read-out design for the large area scintillator detectors: providing good amplitude and time resolutions, Sept 2019. arXiv:1909.01184.

[7] Rojas, Solangel, The Forward Diffractive Detector for ALICE, PoS: vol. LHCP2020, p. 221, 2021. doi.org/10.22323/1.382.0221 .

[8] Broz, M. et al., Performance of ALICE AD modules in the CERN PS test beam JINST 16 no. 01 P01017 (2021), doi.org/10.1088/1748-0221/16/01/P01017.

[9] ALICE Collaboration, Technical Design Report for the Muon Forward Tracker, CERNLHCC-2015-001, ALICE-TDR-018, (2015), https://cds . cern. ch/record/1981898 